\title{
An experience with da Vinci Xi robot-assisted hysterec- tomy for endometrial cancer
}

\author{
Young-Han Park ${ }^{1}$, Min Hyeon Baek ${ }^{1}$, Min Son Kyoung ${ }^{2}$ \\ ${ }^{1}$ Department of Obstetrics and Gynecology, Hallym University Sacred Heart Hospital, Anyang; ${ }^{2}$ Department of Obstetrics and Gynecology, Hallym \\ University Sacred Dongtan Hospital, Hwaseong, Korea
}

Objective: The purpose of this study was to report our experience with the application of the da Vinci Xi system in endometrial cancer.

Methods: The electronic medical records of 21 patients with endometrial cancer who visited Hallym University Sacred Heart Hospital and Hallym University Sacred Dongtan Hospital between 2015 and 2018 were reviewed. Da Vinci Xi robotassisted hysterectomy and pelvic lymph node dissections were performed.

Results: The cancer stages according to the International Federation of Gynecology and Obstetrics staging system were la, $\mathrm{Ib}$, IIl b, and IVb in eight, 11, one, and one patient, respectively. The histology was endometrioid cancer in 19 patients, clear cell carcinoma in one, and serous carcinoma in one. The operation and docking times decreased with an increasing number of cases. The length of hospital stay was not shortened. The operation results were adequate in terms of pathological margin-free status and number of lymph nodes harvested. The bleeding amount was minimal.

Conclusion: The da Vinci $\mathrm{X}$ system is useful as a traditional robotic system or laparoscopic surgical tool in operations for endometrial cancer.

Key Words: Endometrial cancer; Robot assisted radical hysterectomy

\section{INTRODUCTION}

Endometrial cancer has an increasing incidence in Korea and is one of the surgically curable cancers [1]. Vaginal bleeding is the typical and important sign that makes endometrial cancer detectable in its early stage. Early stage endometrial cancer is adequate for minimally invasive surgery, which is known to be a better method than laparoscopy or open laparotomy for endometrial cancer staging [2,3]. The
Food and Drug Administration (FDA) approved the use of the robotic platform for laparoscopic surgery for gynecological cancer hysterectomy in 2005. The FDA warned about the safety problem of the robot-assisted surgical device for mastectomy and other cancers on February 28, 2018. The treatment of the cervical cancer is now controversial, because the surgery using robotic system in locally advanced cervical cancer is not recommended in cervical cancer by FDA [4].

- Received: May 2, 2019 • Revised: October 16, 2019 •Accepted: October 18, 2019

- Correspondening author: Young-Han Park

Department of Obstetrics and Gynecology, Hallym University Sacred Heart Hospital, 22 Gwanpyeong-ro 170beon-gil, Dongan-gu, Anyang 14068, Korea E-mail: lovenabor@naver.com

This is an Open Access article distributed under the terms of the Creative Commons Attribution Non-Commercial License (http://creativecommons.org/ licenses/by-nc/4.0) which permits unrestricted non-commercial use, distribution, and reproduction in any medium, provided the original work is properly cited. 
The robotic platform is developed day bay day. da Vinci Si was the prototype of the robotic surgical system. It has four large arms, which limited its motion and required a large space to maneuver. Docking of the da Vinci Si system is difficult compared with that of the new prototype. Recently, the latest system, da Vinci Xi, was introduced in some cancer and benign tumor surgeries. It is easy to dock because of its movable arm head and joints that can be manipulated to several directions. The length of its arms is enough to approach the deep pelvic cavity from the supraumbilical ports.

Until now, robotic surgery remains a safe treatment method for endometrial cancer. Here, we report our shortterm experience of using the recent model (da Vinci Xi system) in 21 endometrial cancer staging operations.

\section{MATERIALS AND METHODS}

From 2015 to 2018, 21 patients with endometrial cancer were treated with da Vinci Xi system in Hallym University

Table 1. Patients' characteristics

\begin{tabular}{lc}
\hline Characteristic & Case $(\mathbf{n = 2 1 )}$ \\
\hline Age (years) & $58.31 \pm 7.95$ \\
Parity & $1.67 \pm 0.86$ \\
Body mass index $\left(\mathrm{kg} / \mathrm{m}^{2}\right)$ & $25.75 \pm 5.34$ \\
History of abdominal surgery & $12(57.1)$ \\
Comorbid medical disease & $15(71.4)$ \\
Preoperative CA125 level $(\mathrm{IU} / \mathrm{mL})$ & $27.46 \pm 34.99$ \\
\hline
\end{tabular}

Values are presented as mean \pm standard deviation or number (\%).
Sacred Heart Hospital and Hallym University Sacred Dongtan Hospital. Their medical records were reviewed retrospectively in patients who were performed complete surgical staging including pelvic lymph node dissection and hysterectomy. Eleven patients who got simple hysterectomy without lymph node dissection were excluded from the study in order to gain the even operation factors.

\section{RESULTS}

Most patients had early stage la and Ib endometrial cancer. One patient had stage IIIb with pelvic lymph node metastasis, and another had stage IVb with supraclavicular lymph node metastasis. The mean age and body mass index of the patients were 58.3 years and $25.7 \mathrm{~kg} / \mathrm{m}^{2}$, respectively. Fifteen patients had a history of abdominal surgery, with a mean preoperative CA125 level of $27.4 \pm 34.9 \mathrm{U} / \mathrm{mL}$. Endometrioid type was the most frequent histology. One patient had clear cell type, and another had serous carcinoma. The average number of harvested lymph nodes was 44.5 (range, 8-59). None of the patients had conversion to laparotomy and intraoperative or postoperative complications (Table 1).

The mean docking time was 8 minutes. The docking duration was also 8 minutes (range, 6-20 minutes). It is much shorter than the 20-minute docking duration of the previous model (da Vinci SI system) (Fig. 1). The bleeding amount was minimal, and the decrease in hemoglobin level was as small as 50-300 mL, smaller than that in open laparotomy (1,000 mL). The length of hospital stay was long (mean, 10 days), but was shortened to 3 days for those who wanted

\section{Operation time}

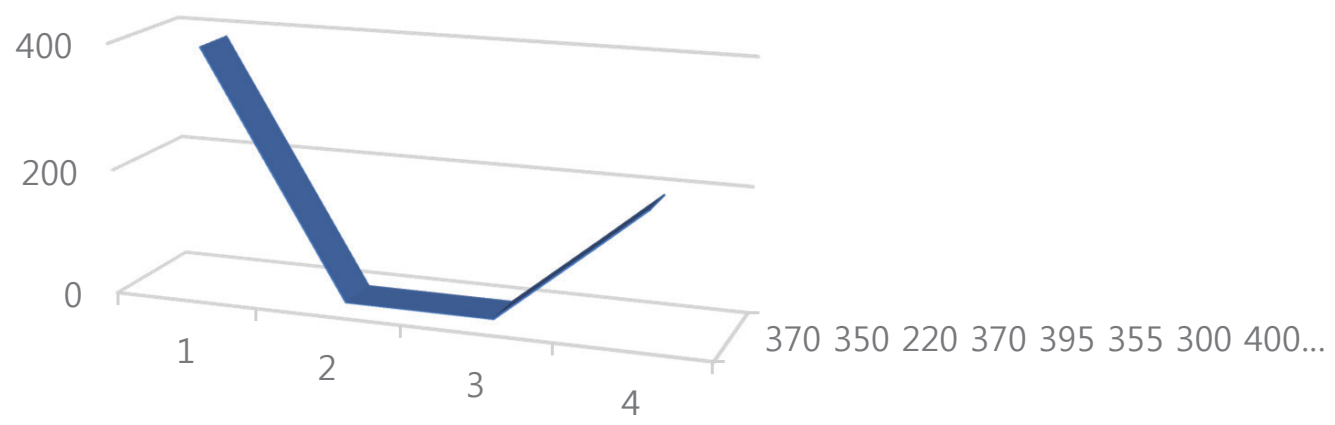

Fig. 1. The trend shows a shortening of operation time with increased experience. 
Table 2. Results of da Vinci Xi radical hysterectomy in patients with endometrial cancer

\begin{tabular}{lc}
\hline & Results $(\mathbf{n}=\mathbf{2 1})$ \\
\hline Docking time (minutes) & $8(6-20)$ \\
Operation time (minutes) & $292.5(190-390)$ \\
Intraoperative complications & None \\
Conversion to laparotomy & None \\
Postoperative complications & None \\
Number of pelvic lymph nodes & $44.5(8-59)$ \\
\hline
\end{tabular}

to be discharged early. The complications of the operation, not the patient's will, prolonged the hospitalization duration. After 3 days, patients are usually free from postoperative pain (Table 2).

\section{DISCUSSION}

Robotic surgery had to be discarded because of the FDA warning issued early in 2019. The danger of robotic surgery is limited to cervical cancer among the gynecological cancers. In endometrial cancer, robotic surgery has a longer history than in cervical cancer and has proven safe. Boggess reported no significant difference in endometrial cancer staging among the three types of surgery, namely laparoscopy, laparotomy, and robotic surgery [3]. In 138 open laparotomy, 81 laparoscopy, and 103 da Vinci robotic hysterectomy cases, robotic surgery showed no inferiority in performance during the operations in terms of incidence of complications, amount of blood loss, and number of pelvic lymph nodes harvested. Park et al. [2] also reported through a meta-analysis, except for vaginal cuff dehiscence, that robotic hysterectomy was better than open laparotomy or laparoscopic surgery in terms of amount of blood loss, length of hospital stay, number of pelvic lymph nodes harvested, and transfusion requirement. According to Walker's report, the survival outcome of laparoscopy is not inferior to that of open laparotomy in endometrial cancer [5]. Patient quality of life is better and recovery is earlier by at least 2 weeks with laparoscopy than with open laparotomy [6]. The rapid recovery from operative discomfort with robotic surgery is undeniable. Endometrial cancer is a good candidate for robotic minimally invasive surgery. Thus, if available, robotic minimally invasive surgery should be introduced in patients with endometrial cancer.

In the lithotomy position after anesthesia, docking of the da Vinci Xi system is started. The docking time is measured from the time of positioning the patient to the starting point of the operation in the console box. This is the benefit of the Xi system because of its movable arm head. In docking, a green laser light guides the head to the supraumbilical port. The lean arms of the da Vinci Xi system are easy to move and could be manipulated toward the correct direction easily.

As endometrial cancer is surrounded by thick and strong myometrium, the uterus is safe to operate and manipulate during operation. Before the cutting of the round ligament, the bilateral tubes are usually coagulated with bipolar forceps to protect the spillage of endometrial cancer cells into the peritoneal cavity. The balloon of the uterine cavity is dilated at this point after the tubal cauterization. The other procedures are the same as those in radical hysterectomy. Bilateral external iliac, internal iliac, and obturator lymph node dissections are performed serially. The para-aortic lymph nodes are usually dissected to the infra-mesenteric artery level. One patient had para-aortic lymph node-positive cancer stage IVb with confirmed left supraclavicular metastasis. Owing to the limited coverage field of magnetic resonance imaging, the para-aortic lymph node metastasis was missed preoperatively. After the para-aortic metastasis was confirmed with biopsy, positron-emission tomography and computed tomography was performed, and fine-needle aspiration biopsy disclosed a distant metastasis. Even in the patients with stage IVb cancer, the hysterectomy margin was tumor-free pathologically, which means that the hysterectomy was adequate in all of the patients with endometrial cancer. It was also due to the longer-arm instruments of the Xi system, which can approach the deep pelvic cavity without changing the machine docking pattern.

The number of harvested lymph nodes was 45.5 (range, 8-59), which is not inferior to that in open laparotomy and laparoscopic surgery, and in other reports $[3,4,6]$.

A robotic system is a machine without intelligence that is used by surgeons. The surgeon is the determinant of the operation results. Surgeon skill is a highly important factor that influences the results in clinical trials. The uterine manipulator and operation procedure could be the co-factors that influence the results of the treatment of cervical cancer. However, in endometrial cancer, surgery seems effective and acceptable results can be expected. 
In conclusion, surgery with the da Vinci Xi system is recommended as the primary operation for patients with endometrial cancer. The da Vinci Xi system is easy and safe to use in endometrial cancer surgery. It is a much-improved machine compared with the old version, the Si system. The next-generation robot system, which includes a more advanced single-port robotic system, is much awaited. Further studies with prospective randomization and larger number of patients are necessary.

\section{Conflict of interest}

No potential conflict of interest relevant to this article was reported.

\section{References}

1. National Cancer Information Center. The cancer incidence according to the organs [Internet]. Goyang: National Cancer Information Center; c2019 [cited 2019 Oct 24]. Available from:
https://www.cancer.go.kr/lay1/S1T639C641/contents.do.

2. Park DA, Lee DH, Kim SW, Lee SH. Comparative safety and effectiveness of robot-assisted laparoscopic hysterectomy versus conventional laparoscopy and laparotomy for endometrial cancer: a systematic review and meta-analysis. Eur J Surg Oncol 2016;42:1303-14.

3. Boggess JF, Gehrig PA, Cantrell L, Shafer A, Ridgway M, Skinner EN, et al. A comparative study of 3 surgical methods for hysterectomy with staging for endometrial cancer: robotic assistance, laparoscopy, laparotomy. Am J Obstet Gynecol 2008;199:360.e1-9.

4. Ramirez PT, Frumovitz M, Pareja R, Lopez A, Vieira M, Ribeiro $\mathrm{R}$, et al. Minimally invasive versus abdominal hysterectomy for cervical cancer. N Engl J Med 2018;379:1895-904.

5. Walker JL, Piedmonte MR, Spirtos NM, Eisenkop SM, Schlaerth JB, Mannel RS, et al. Laparoscopy compared with laparotomy for comprehensive surgical staging of uterine cancer: gynecologic oncology group study LAP2. J Clin Oncol 2009;27:5331-6.

6. Lundin ES, Wodlin NB, Nilsson L, Kjölhede P. A prospective randomized assessment of quality of life between open and robotic hysterectomy in early endometrial cancer. Int J Gynecol Cancer 2019 Mar 28 [Epub]. http://dx.doi.org/10.1136/ijgc2019-000285. 